\title{
Immune response to rabbit coccidiosis: a comparison between infections with Eimeria flavescens and $E$. intestinalis
}

\author{
Michal Pakandl ${ }^{1}$, Lenka Hlásková ${ }^{1}$, Martin Poplštein ${ }^{2}$, Marie Nevečeřalováa ${ }^{3}$, Tomáš Vodička ${ }^{3}$, Jiří Salát $^{1}$ and \\ Jitka Mucksová ${ }^{2}$ \\ ${ }^{1}$ Institute of Parasitology, Biology Centre, Academy of Sciences of the Czech Republic, Branišovská 31, 37005 České \\ Budějovice, Czech Republic; \\ ${ }^{2}$ BIOPHARM, Research Institute of Biopharmacy and Veterinary Drugs, a.s., Pohoří-Chotouň, 25449 Jílové u Prahy, Czech \\ Republic; \\ ${ }^{3}$ Faculty of Science, University of South Bohemia, Branišovská 31, 37005 České Budějovice, Czech Republic
}

Key words: rabbit coccidiosis, Eimeria intestinalis, Eimeria flavescens, immune response, ELISA, lymphocyte proliferation, intraepithelial lymphocytes

\begin{abstract}
Seven- to eight-week-old rabbits were infected with Eimeria intestinalis Cheissin, 1948, a highly immunogenic coccidium, or Eimeria flavescens Marotel et Guilhon, 1941, which is weakly immunogenic. Immune response was investigated at 7 , 14 and 21 days post inoculation (DPI). The level of serum immunoglobulins, lymphocyte proliferation stimulated by parasite antigens and weight of mesenteric lymph nodes (MLN) showed similar dynamics in rabbits inoculated with both coccidia species. The amount of serum IgG and IgM, but not IgA, was increased from 14 DPI. The lymphocytes from MLN of infected animals significantly reacted to stimulation with parasite antigen 14 and 21 DPI and MLN were enlarged at 14 DPI. Thus, both parasite species elicited immune response characterized by these parameters in a similar manner despite of their different immunogenicity. The only apparent difference in the responses was in the percentage of CD8+ lymphocytes in the specific site of parasite development (the last third of the small intestine in E. intestinalis, caecum in E. flavescens), which increased in rabbits infected with $E$. intestinalis but not with $E$. flavescens. This parameter reflects the status of local immunity and hence the results suggest that the local reaction plays an important role in induction of protective immunity to coccidia in rabbits.
\end{abstract}

Coccidiosis causes important economic losses in livestock and poultry production in domestic animals including rabbits. The most pathogenic species of rabbit coccidia are Eimeria flavescens Marotel et Guilhon, 1941 and Eimeria intestinalis Cheissin, 1948. However, these species markedly differ in their immunogenicity. While E. intestinalis is highly immunogenic, and even infection with as few as 6 oocysts can initiate significant immunity (Coudert et al. 1993), E. flavescens is weakly immunogenic (Norton et al. 1979). Although infection with coccidia in chickens, mice and other hosts induces humoral response, numerous studies have demonstrated a major role of cell-mediated immunity (reviewed by Wakelin and Rose 1990, Lillehoj 1998 and Lillehoj et al. 2004), in which both CD4+ and CD8+ lymphocytes are involved. We have compared the immune reaction induced by two coccidia using three methods: ELISA to study humoral response, a lymphocyte proliferation test to evaluate cell-mediated immunity, and flow cytometry analysis to study changes in the CD4+ and CD8+ composition in mesenteric lymph nodes (MLN), as well as measuring intraepithelial lymphocytes (IEL). The latter parameter particularly reflects local immunity, which is very important in host defence against coccidia. The weight of the MLN and spleen was also determined.

\section{MATERIALS AND METHODS}

Animals

SPF New Zealand white rabbits, weight category $1-1.5 \mathrm{~kg}$ (corresponding to 7-8 weeks of age), originating from Charles River, Germany, were provided by AnLab, Ltd., Prague.

\section{Parasites}

Two parasite species were used: E. flavescens, a strain isolated in our laboratory, and E. intestinalis, kindly provided by Pierre Coudert (INRA, BASE, Tours, France). Only freshly sporulated oocysts (1-40 days after sporulation) were used to inoculate experimental animals.

\section{Preparation of soluble antigens}

Oocyst antigen. Purified oocysts were vigorously shaken with glass beads (diameter $1 \mathrm{~mm}$ ) using a beadbeater, sonicated and centrifuged at $6,000 \mathrm{~g}$ for $5 \mathrm{~min}$. The protein concentration was assessed and the supernatant was divided into aliquots and frozen.

Sporozoite antigen. Oocysts were broken in a homogenizer and obtained sporocysts were treated for $3.5 \mathrm{~min}$ with excystation medium composed of bile salts (Sigma) $1 \%$ and trypsin (Sigma) $0.4 \%$ in PBS (Ceré et al. 1995). Liberated sporozoites were washed with PBS and purified by filtration through Isopore ${ }^{\mathrm{TM}}$ membrane filters with pores 3 or $5 \mu \mathrm{m}$ in diameter. The antigen was prepared by four cycles of freezing in liquid nitrogen, thawing and sonication. 
Antigen for ELISA. The antigen for ELISA was prepared basically by the same method as sporozoite antigen. Purified oocysts were freezed/thawed and sonicated four times. After centrifugation at $6,000 \mathrm{~g}$ for $5 \mathrm{~min}$, the supernatant was collected and frozen.

\section{Enzyme linked immunosorbent assay (ELISA)}

Soluble oocyst antigens from E. flavescens and E. intestinalis were used. The 3590 High Binding 96-well polystyrene microtitre plates (Costar, USA) were coated with $10.0 \mu \mathrm{g} / \mathrm{ml}$ parasite antigen in $0.05 \mathrm{M}$ carbonate buffer ( $\mathrm{pH}$ 9.6) and incubated for $18 \mathrm{~h}$ at $4^{\circ} \mathrm{C}$. The plates were washed three times with PBS supplemented with $0.5 \%$ Tween 20 (PBS-Tw) and then blocked with $2 \%$ skim milk (Fluka) in PBS for $1 \mathrm{~h}$ at $37^{\circ} \mathrm{C}$. The sera were finally diluted $1: 160$ with $1.0 \%$ BSA in PBS. A $100 \mu 1$ volume of each sample was applied to the microtitre plate wells and incubated for $2 \mathrm{~h}$ at $37^{\circ} \mathrm{C}$. Peroxidase-labeled goat anti-rabbit IgG ab-6721-1, goat anti-rabbit IgM ab-2755-500, or goat anti-rabbit IgA ab-2759-500 (Abcam, UK) were diluted 1:5,000 with 1\% BSA in PBS-Tw and added in $100 \mu \mathrm{l}$ volumes to the appropriate wells and the plates were incubated for $1 \mathrm{~h}$ at $37^{\circ} \mathrm{C}$. Citric acid $(0.04 \mathrm{M})$ in phosphate buffer ( $\mathrm{pH} 5.0$ ), containing orthophenylenediamine P-6912 (Sigma) $0.05 \%$ and hydrogen peroxide $0.1 \%$, was added in $100 \mu 1$ volumes per well. The reaction was stopped after $15 \mathrm{~min}$ by adding $100 \mu \mathrm{l}$ of $2 \mathrm{M}$ sulphuric acid. Absorbance was read at $492 \mathrm{~nm}$ on the Hidex Chameleon microELISA reader, Driver Version 4.20 (Hidex, Finland). The ratio A/A0 [absorbance of the samples/absorbance of blank (PBS)] was then calculated.

\section{Preparation of lymphocytes for flow cytometry}

The MLN were crushed on a mesh using a syringe piston. The cells were then suspended in RPMI 1640 medium containing 2\% of FetalClone I (HyClone). The cell suspension was then washed four times in the same medium. Finally, the cells were placed in PBS supplemented with $1 \%$ of FetalClone I. The cells were then labeled for flow cytometry.

The IEL were isolated from the sites specific for the two coccidia, i.e. the last third of the small intestine in the case of E. intestinalis and caecum for E. flavescens. The IEL from the small intestine were isolated using modified method of Todd et al. (1999). The intestine was dissected and cut into several segments. The Peyer's patches were removed and the intestinal content was flushed out with PBS using a syringe inserted into the lumen. The PBS was then replaced by RPMI 1640 medium containing $2 \%$ of FetalClone I and the tissue was incubated at $4{ }^{\circ} \mathrm{C}$ for $1.5 \mathrm{~h}$. These conditions are unfavourable for enterocytes, while lymphocytes survive this incubation undamaged. The lumen was then gently flushed with Hank's Balanced Salt Solution (HBSS) containing 5\% of FetalClone I and $1 \mathrm{mM}$ of dithioerythritol at $37^{\circ} \mathrm{C}$, and the content of the lumen was squeezed out by manual compression. This procedure was repeated 5 times and the intestinal eluate was saved, filtered through rough nylon wool and decanted for $10 \mathrm{~min}$ to remove debris and cell aggregates. The cells were collected by centrifugation, resuspended in 30\% Percoll (Sigma) mixed with medium and centrifuged at room temperature at $350 \mathrm{~g}$ for $15 \mathrm{~min}$. The sediment was resuspended in $45 \%$ Percoll solution and $75 \%$ Percoll solution was layered under the suspension. After centrifugation for $30 \mathrm{~min}$ at $350 \mathrm{~g}$, viable IEL were collected from the interface between two layers.
To obtain IEL from the caecum, this part of the intestine was dissected, opened, thoroughly washed in PBS, cut into segments about $3 \mathrm{~cm}$ long and placed for $1.5 \mathrm{~h}$ in cold medium. It was then moderately shaken in HBSS containing 5\% of FetalClone I for $30 \mathrm{~min}$ at $37^{\circ} \mathrm{C}$. The subsequent isolation steps were performed as for the cells from the small intestine.

\section{Flow cytometry}

The cells obtained from MLN and intestine were incubated at $4^{\circ} \mathrm{C}$ with monoclonal antibodies (Serotec, UK) directed to rabbit CD4 (MCA799) or CD8 (MCA1576), as well as with mice IgG2a (isotype control to anti-CD4, MCA929) and IgG1 (isotype control to CD8, MCA928). Monoclonal antibodies were diluted 100 times. The second step was incubation with fluorescein-isothiocyanate (FITC)-conjugated goat anti-mouse immunoglobulins (Sigma). We also used controls in which no primary antibodies were added and the cells were then incubated with or without FITC conjugate. The percentage of CD4 or CD8 positive cells was assessed by the Coulter XL-MC flow cytometer. The percentage of cells non-specifically labeled with isotype controls (usually less than $1.5 \%$ ) was subtracted from the values obtained with anti-CD4 or anti-CD8 monoclonal antibodies.

\section{Lymphocyte proliferation test}

The lymphocytes were recovered from aseptically removed MLN as described above, counted and their viability was tested by Trypan blue exclusion. The cells were suspended in RPMI 1640 medium containing 10\% bovine foetal serum, 200 $\mathrm{mM}$ L-glutamine, $100 \mathrm{U} / \mathrm{ml}$ penicillin, $10 \mu \mathrm{g} / \mathrm{ml}$ streptomycin, and $5 \times 10^{-5} \mathrm{M}$ mercaptoethanol and seeded into 96-well flatbottom microtitre plates (TPP) $\left(10^{6}\right.$ cells $/ 200 \mu 1$ per well). To test the stimulation, $5 \mu \mathrm{g} / \mathrm{ml}$ of the mitogens concanavalin A, phytohaemagglutinin (non-specific stimulation) or soluble antigens (prepared from both oocysts and sporozoites) in concentrations $10,5,1$ and $0.2 \mu \mathrm{g} / \mathrm{ml}$ were added into individual wells. Four wells were used for each specific antigen and concentration. The cells were cultivated in a humified $\mathrm{CO}_{2}$ incubator $\left(5 \% \mathrm{CO}_{2}, 37^{\circ} \mathrm{C}\right)$ and proliferation was then assessed by Cell-Counting Kit 8 (Fluka). The cells were transferred in another flat-bottom plate $(100 \mu 1 /$ well $)$ and incubated with 10 $\mu 1$ of the reagent for $4 \mathrm{~h}$. The absorbance at $450 \mathrm{~nm}$ was then measured. The intensity of stimulation was expressed as a stimulation index (SI), which was calculated as the ratio of the highest absorbance (usually in wells with concentration $5 \mu 1$ antigen $/ \mathrm{ml}$ ) to the absorbance in wells in which no antigen was added as a control. Stimulation by mitogens alone served as a criterion that the conditions of cultivation are favourable for lymphocyte proliferation and only the results with parasite antigen are shown.

\section{Experimental design}

A total of 30 rabbits were inoculated with 2,000 oocysts of either E. intestinalis or E. flavescens and sacrificed at various intervals: 7, 14 and 21 days post-inoculation (DPI). Five animals were killed for each interval and parasite species. Another group of 10 animals served as an uninfected control. The intestine (ileum in rabbits infected with $E$. intestinalis, caecum in those infected with E. flavescens, and both organs in control animals) and MLN were removed and the cells from these organs were processed using the methods described above. The spleens and the whole MLN from each animal were weighed and the ratio of weight of the organ/total body weight 
was determined. Blood samples were taken in control animals at the day of slaughter and in the infected animals at the day of inoculation (0 DPI) and after 7, 14 and 21 days. A volume of $60 \mu 1$ of blood was taken from the ear vein, diluted ten times with PBS and the serum was frozen until examined by ELISA. The sera from control animals were examined using both $E$. intestinalis and E. flavescens antigens, while the sera from infected rabbits were tested only with antigen from the given species. To study the dynamics of antibody response, only the values obtained in control group and in the group of rabbits sacrificed at 21 DPI were statistically evaluated, as the animals from other groups were sacrificed earlier.

\section{Statistical analysis}

The statistical evaluation was based on Tukey's HSD test at $\mathrm{P} \leq 0.05$ and performed using the application ANOVA, which is included in the Statistica.cz software.

\section{RESULTS}

\section{Humoral response}

Antibody response was induced in the rabbits inoculated both with E. intestinalis and E. flavescens. The values of $\operatorname{IgG}$ and $\operatorname{IgM}$ antibodies were higher in $E$. flavescens at 14 and $21 \mathrm{DPI}$. The results are shown in Figs. 1 and 2. After infection with both coccidia, the level of IgG increased from 14 DPI onwards. The highest concentration of $\operatorname{IgM}$ antibodies was observed at 14 DPI, but the value did not differ significantly from that found at 21 DPI. However, the value assessed at 14 DPI differed from that in control animals as well as from the values at 0 and 7 DPI. The mean level of IgA antibodies was slightly enhanced at 14 and 21 DPI, but the differences were not significant. Generally, no differences in the antibodies of all three isotypes were found between the samples taken at 0 and 7 DPI, as well as from control group and even between animals sacrificed 14 and 21 DPI.

\section{Weight of spleen and MLN}

No differences in weight of the spleen were found in rabbits infected with E. intestinalis (Fig. 3). In contrast, 14 and 21 DPI the spleens were enlarged after infection with $E$. flavescens. A significantly higher weight of the MLN was noted 14 DPI in rabbits infected both with $E$. intestinalis and E. flavescens.

\section{Dynamics of CD4+ and CD8+ $T$ cell subpopulations in IEL and MLN}

No significant differences were observed in the percentage of CD4+ and CD8+ lymphocytes in the MLN from rabbits inoculated with either $E$. intestinalis or $E$. flavescens (Figs. 4, 5). In E. flavescens, the proportion of CD4+ and CD8+ lymphocytes among the IEL from the caecum also remained unchanged during the experiment. The only significant change was a higher percentage of CD8+ lymphocytes in the IEL of rabbits infected with $E$. intestinalis at 14 DPI (61\%). This value was $38 \%$ in the control group and $42 \%$ and $52 \%$ in the rabbits sacrificed at 7 and $21 \mathrm{DPI}$, respectively. At 21

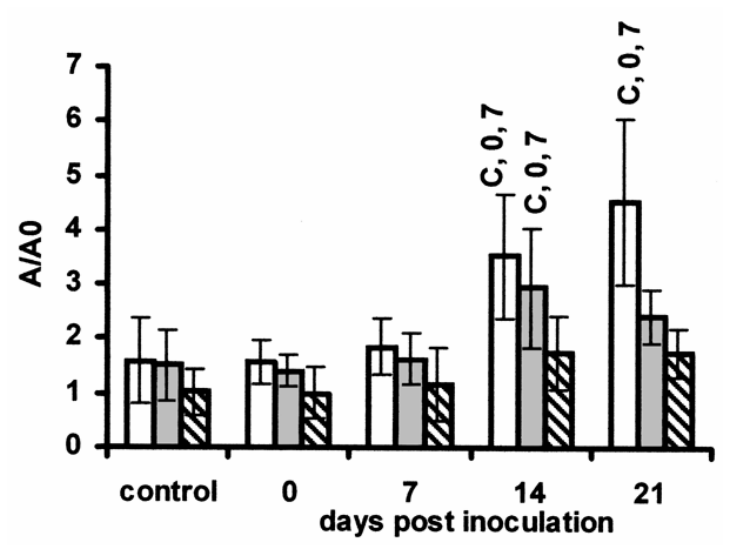

Fig. 1. Levels of antibodies assessed by ELISA in rabbits infected with Eimeria intestinalis. Statistically significant differences are indicated above the columns ( $\mathrm{C}-$ control; 0,7 - DPI 0 and 7). A/A0 - absorbance of the samples/absorbance of blank (PBS). The blood samples were taken at the days post inoculation as indicated. The columns represent the mean value obtained from five animals. $\square_{\mathrm{IgG}}, \square \mathrm{IgM}, \boldsymbol{\nabla}_{\mathrm{IgA}}$.

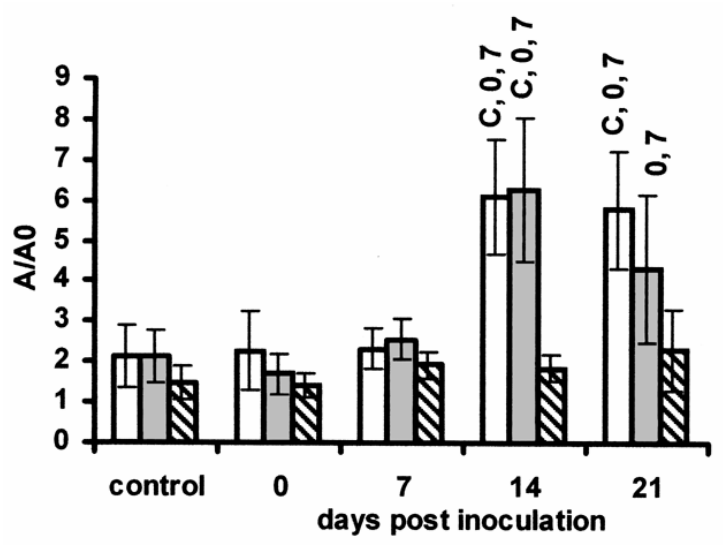

Fig. 2. Levels of antibodies assessed by ELISA in rabbits infected with Eimeria flavescens. The sampling was the same as in rabbits infected with E. intestinalis. For explanation of abbreviations and rasters of columns see Fig. 1.

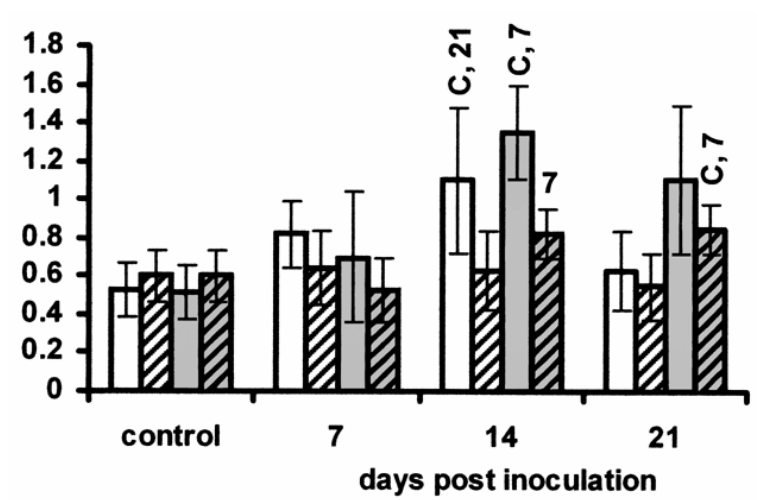

Fig. 3. Weights of mesenteric lymph nodes (MLN) and spleen [per mille of total body weight] in experimentally infected rabbits. All the MLN from each rabbit were weighed. $\square$ infected with Eimeria intestinalis, MLN; $\square$ infected with $E$. intestinalis, spleen; $\square$ infected with E. flavescens, MLN; $\square$ infected with E. flavescens, spleen. 


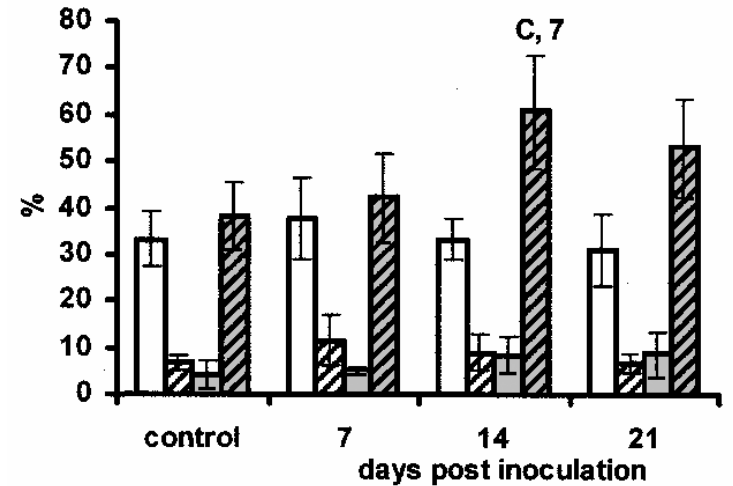

Fig. 4. Percentage of CD4+ and CD8+ T-cell subpopulations in rabbits infected with Eimeria intestinalis. The only significant difference was found in the proportion of CD8+ cells 14 days post inoculation, when the value differed from that at the control group and the group examined 7 days post inoculation.

CD4+ cells in mesenteric lymph nodes (MLN), $\square$ CD8+ cells in MLN, $\square$ CD4+ cells in intraepithelial lymphocytes (IEL), $\boldsymbol{D}$ CD8+ cells in IEL.

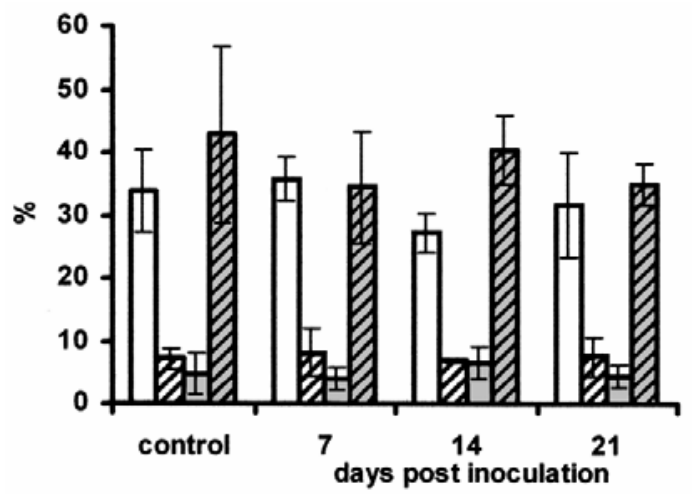

Fig. 5. Percentage of CD4+ and CD8+ T-cell subpopulations in rabbits infected with Eimeria flavescens. The columns are distinguished as in Fig. 4. No significant difference was found at any day post inoculation.

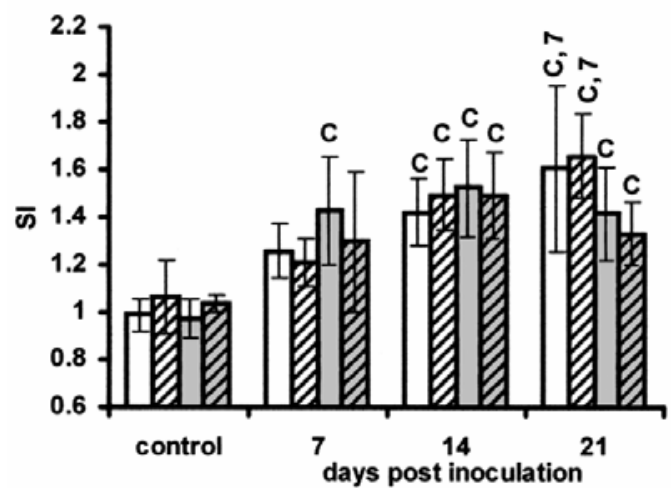

Fig. 6. Stimulation indexes (SI) in cultivated lymphocytes from rabbits inoculated with Eimeria intestinalis or E. flavescens, respectively. The cells were incubated with homologous antigens. The SI was calculated as the ratio of absorbance at $450 \mathrm{~nm}$ after incubation for 4 hours with Cell-Counting Kit 8 in wells with- to without antigen. $\square$ oocyst antigen from $E$. intestinalis, $\square$ sporozoite antigen from E. intestinalis, oocyst antigen from $E$. flavescens, $\boldsymbol{Z}$ sporozoite antigen from E. flavescens.
DPI, the percentage of CD8+ was also higher compared to the control, but statistically this value did not differ from that in the other groups.

\section{Lymphocyte proliferation test}

The proliferation of lymphocytes from the MLN of infected rabbits was stimulated by a specific antigen. The SI increased from 7 DPI and the difference became significant in relation to the control groups from 14 DPI onwards, as in the case of stimulation by oocyst antigen from $E$. flavescens at 7 DPI (Fig. 6). Moreover, the SI in cells of rabbits inoculated with E. intestinalis and killed 21 DPI differed from that in the group investigated at 7 DPI as well. Very similar results were obtained in all cases using either the oocyst or sporozoite antigen. The highest SI was noted 14 DPI in rabbits infected with $E$. flavescens (1.50 for both antigens) and 21 DPI after infection with E. intestinalis (1.61 for oocyst antigen, 1.66 for sporozoite antigen). The lymphocytes cultivated in the presence of the mitogens served as a positive control. Cells from rabbits inoculated with both coccidian species, as well as from control animals, were stimulated (data not shown).

\section{DISCUSSION}

There are very little data concerning the immune response of rabbits to coccidia. The most detailed study was performed by Renaux et al. (2003), who worked with the highly immunogenic Eimeria intestinalis. Using a lymphocyte proliferation test, flow cytometry analysis and ELISA, they observed a marked reaction to infection. The immunogenicity of E. intestinalis was assessed by Coudert et al. (1993) measuring oocyst production and weight gains after secondary infection. The same method showed that E. flavescens is weakly immunogenic (Norton et al. 1979), and this was confirmed even with the isolate used in the present report (unpublished results). The aim of this study was to compare the immune response after infection with two highly pathogenic coccidia, which markedly differ in their immunogenicity.

Concerning $E$. intestinalis, our results are in some regards different from those of Renaux et al. (2003), especially by higher proportions of CD4+ and CD8+ cells in the cells isolated from the small intestine in our experiments. In our opinion, this depends on the method of isolation of IEL and the definition of the gate in which the cells are counted by the flow cytometer. Moreover, we used a method optimised for the isolation of IEL, while Renaux et al. (2003) isolated the cells from scraped mucosa, thus working with cells from both the epithelium and lamina propria. This may explain the discrepancies between our and their results.

In our study, the amount of serum IgG, percentage of CD8+ lymphocytes in the ileum, SI value and weight of MLN increased at 14 DPI. In addition to this, Renaux et al. (2003) observed a significant increase in the percent- 
age of CD8+ lymphocytes in the MLN 14 and 21 DPI, as well as CD4+ cells in the ileum 14 DPI. In our experiments, the percentage of CD8+ cells in MLN was $6.8,11.6,8.9$ and 6.9 in the control group and at 7, 14, and 21 DPI, respectively. However, these values did not differ significantly and even the average values were not so dramatically changed, as was reported by Renaux et al. (2003). This aspect is also important in terms of the percentage of CD4+ lymphocytes found in the ileum, where our values $(4.3,5.0,8.4$ and 8.8 in the control group and in the rabbits sacrificed at 7, 14 and 21 DPI, respectively) were also not statistically different using afore mentioned method. Nevertheless, the dynamics of the immune response in our experiments was generally similar to that observed by Renaux et al. (2003).

Although development of specific antibodies is not an essential component of host resistance to coccidia infection, antibodies play a contributory role (Wakelin and Rose 1990). In our experiments, no substantial differences in antibody response were found during infection with the two species of coccidia, regardless of their different immunogenicity. After experimental primary infections of rats with Eimeria nieschulzi (Rose et al. 1984, Smith et al. 1995), the IgM response was rapid and its peak was relatively brief. In contrast, IgG level needed more than 20 days to reach their maximum. Although we sacrificed rabbits $21 \mathrm{DPI}$, and the dynamics of humoral response was not studied after such a longer interval, our results are roughly similar to the previously mentioned data.

Both coccidia species used in our experiments have a prepatent period of nine days. The studies on their life cycles (Norton et al. 1979, Licois et al. 1992, Pakandl et al. 2003) showed that the last generation of meronts is developed at 7 DPI. At 14 DPI, only gamonts and oocysts are present in the intestine and the rabbits excrete oocysts, but their numbers are lower than at 10-12 DPI. The endogenous stages are virtually absent at 21 DPI. The highest percentage of CD8+ lymphocyte in the ileum after infection with $E$. intestinalis and the heaviest MLN in rabbits infected with both $E$. intestinalis and $E$. flavescens, which were observed at 14 DPI, correspond rather to the acute phase of infection. These parameters reflect the proliferation of lymphocytes and their migration towards the specific site of parasite development, and thus local immune response. On the other hand, the levels of serum IgG and IgM antibodies, and the ability of lymphocytes to proliferate after stimulation with specific antigen, persisted after disappearance of the parasites.

Besides lymphocytes from the MLN, we tried to perform a lymphocyte proliferation test with lymphocytes from peripheral blood as well, but their proliferation was markedly lower compared to lymphocytes from MLN (data not shown). Therefore, this parameter was not a useful criterion for an immune response. Renaux et al. (2003) did not find any significant differences in the proliferation of splenocytes stimulated by parasite antigen between control rabbits and those infected with $E$. intestinalis. Unlike the coccidia infection in rabbits, splenocytes and peripheral blood lymphocytes isolated from calves infected with Eimeria bovis were stimulated by parasite antigen (Hermosilla et al. 1999).

Enlargement of the MLN appeared as a response to parasite infection in rabbits infected with both parasite species, despite of their different immunogenicity. Moreover, the spleen was enlarged in the weakly immunogenic E. flavescens, but not in E. intestinalis, which is known as a strong immunogen. In the experiments performed by Renaux et al. (2003), no enlargement of the spleen was observed in rabbits infected with $E$. intestinalis, but the weight of the MLN increased at 14 DPI and this observation is in agreement with our results.

Many authors have shown that IEL play a crucial role in the host immune defence against coccidia. InagakiOhara et al. (2006) recently demonstrated two mechanisms involved in the local reaction: production of cytokines to induce protective immunity and expression of junctional molecules to preserve epithelial barrier. Significantly increased number of CD4+ and CD8+ T lymphocytes were observed in the small intestine of mice after primary infection with Eimeria papillata (Schito et al. 1998), but increasing of CD4+ cells was more conspicuous. Shi et al. (2001) studied early responses $(0,1$, and 2 DPI) to infection with Eimeria separata in rats. CD4+ cells predominated in the intestine of animals after primary infection, whereas CD8+ cells represented the major T-cell subset in challenged rats. Rose et al. (1992) demonstrated in mice, in which CD4+ or CD8+ lymphocytes were depleted, the importance of mechanisms involving the function of CD4+ T lymphocytes in the control of primary infections with Eimeria spp. and their results also indicated that $\mathrm{CD} 8+$ cells play some part in the expression of resistance to reinfection. Taken together, the number of intraepithelial CD4+ lymphocytes increases during primary infection in rodents, whereas CD8+ cell are involved rather in the reaction to challenge.

Hermosilla et al. (1999) found increased portion of CD4+, but not CD8+, lymphocytes in gut-associated lymph nodes of calves infected with $E$. bovis and sacrificed 35 DPI. In peripheral blood lymphocytes, the proportions of both CD4+ and CD8+ cells was transiently enhanced 12 DPI, but declined until 25 DPI and finally reached control values. In contrast to this, Renaux et al. (2003) observed significantly increased proportion of CD8+, but not CD4+ cells in MLN of rabbits infected with $E$. intestinalis. Aleksandersen et al. (1995) observed increased presence of CD8+ lymphocytes in the epithelial areas of distal jejunum of lambs after primary infection with Eimeria ovinoidalis and this corresponds to the results obtained in rabbits (Renaux et al. 2003 and the present work). In conclusion, the dy- 
namics and mechanisms of the immune response to coccidiosis differ in various mammalian hosts.

Although E. flavescens is less immunogenic, the antibody response, enlargement of MLN and lymphocyte proliferation after stimulation with specific antigen were similar to those in rabbits infected with E. intestinalis. The only difference between rabbits infected with these two coccidia species was found in the percentage of CD8+ lymphocytes in IEL from the specific site of parasite development. This parameter was enhanced in rabbits infected with the highly immunogenic species $E$. intestinalis, but not with E. flavescens, suggesting the importance of local immune response in the elicitation of protective immunity against coccidia, as well as a significant role of $\mathrm{CD} 8+$ lymphocytes in this process.
Nevertheless, E. flavescens is also able to elicit immune reactions, which was demonstrated by humoral response, proliferation of lymphocytes stimulated by parasite antigen and enlargement of spleen and MLN. However, primary infection with this species does not lead to a significant suppression of parasite development and weight loss during secondary infection. Perhaps a different immunological memory developed after primary infection with E. intestinalis and E. flavescens, which may also play a role in the reaction to a challenge infection with these coccidia.

Acknowledgements. This work was supported by a grant of the Czech Science Foundation (524/05/2328) and the research project of the Institute of Parasitology (Z60220518). We thank Mrs. Marie Kreimová for technical assistance.

\section{REFERENCES}

AleKsandersen M., LANDSVerk T., GJerde B., Helle O. 1995: Scarcity of gamma-delta T-cells in intestinal epithelia containing coccidia despite general increase of epithelial lymphocytes. Vet. Pathol. 32: 504-512.

Ceré N., LiCOIS D., HumberT J.F. 1995: Study of inter- and intraspecific variation of Eimeria spp. from the rabbit using random amplified polymorphic DNA. Parasitol. Res. 81: 324 328.

COUdert P., Licois D., Provôt F., DROUET-Viard F. 1993: Eimeria sp. from rabbit (Oryctolagus cuniculus): pathogenicity and immunogenicity of Eimeria intestinalis. Parasitol. Res. 79: 186-190.

Hermosilla C., BÜRGer H.-J., ZAHNER H. 1999: T cell responses in calves to a primary Eimeria bovis infection: phenotypical and functional changes. Vet. Parasitol. 84: 49-64.

InAGAKI-OHARA K., DEWI F.N., HisAeda H., SMITH A.L., JiMI F., MiYAHIRA M., ABDEL-AleEM A.S.F., Hori Y., NAWA Y. 2006: Intestinal intraepithelial lymphocytes sustain the epithelia barrier function against Eimeria vermiformis infection. Infect. Immun. 74: 5292-5301.

LiCOIS D., COUderT P., BAHAgIA S., Rossi G.L. 1992: Characterisation of Eimeria species in rabbits (Oryctolagus cuniculus): endogenous development of Eimeria intestinalis Cheissin, 1948. J. Parasitol. 78: 1041-1046.

LILLEHOJ H.S. 1998: Role of T lymphocytes and cytokines in coccidiosis. Int. J. Parasitol. 28: 1071-1081.

LiLlEHOJ H.S., MiN W., DALlOUL R.A. 2004: Recent progress on the cytokine regulation of intestinal immune response to Eimeria. Poultry Sci. 83: 611-623.

NORTON C.C., CATCHPOLE J., JOYNER L.P. 1979: Redescriptions of Eimeria irresidua Kessel \& Jankiewicz, 1931 and E. flavescens Marotel \& Guilhon, 1941 from the domestic rabbit. Parasitology 79: 231-248.
PAKANDL M., ČERNíK F., COUDERT P. 2003: The rabbit coccidium Eimeria flavescens Marotel and Guilhon, 1941: an electron microscopic study of its life cycle. Parasitol. Res. 91: 304-311.

RenauX S., Quéré P., BuZoni-Gatel D., SEWALd B., LE Vern Y., COUdERT P., DROUET-VIARD F. 2003: Dynamics and responsiveness of T-lymphocytes in secondary lymphoid organs of rabbits developing immunity to Eimeria intestinalis. Vet. Parasitol. 110: 181-195.

ROSE M.E., HESKETH P., WAKELIN D. 1992: Immune control of murine coccidiosis - CD4+ and CD8+ lymphocytes- $\mathrm{T}$ contribute differentially in resistance to primary and secondary infections. Parasitology 105: 349-354.

Rose M.E., PePPARD J.V., HobBS S.M. 1984: Coccidiosis: characterization of antibody responses to infection with Eimeria nieschulzi. Parasite Immunol. 6: 1-12.

SCHITO M.L., CHOBOTAR B., BARTA J.R. 1998: Cellular dynamics and cytokine responses in BALB/c mice infected with Eimeria papillata during primary and secondary infections. J. Parasitol. 84: 328-337.

SHI M.Q., HUTHER S., BURKHART E., ZAHNER H. 2001: Lymphocyte subpopulations in the caecum mucosa of rats after infection with Eimeria separata: early responses in naïve and immune animals to primary and challenge infections. Int. J. Parasitol. 31: 49-55.

SMith N.C., OVInGton K.S., DePlazes P., ECKERT J. 1995: Cytokine and immunoglobulin subclass responses of rats to infection with Eimeria nieschulzi. Parasitology 111: 51-57.

TODD D., Singh A.J., Greiner D.L., Mordes J.P., Rossini A.A., BORTELL R. 1999: A new isolation method for rat intraepithelial lymphocytes. J. Immunol. Methods 224: 111-127.

WAKELIN D., RosE M.E. 1990: Immunity to coccidiosis. In: P.L Long (Ed.), Coccidiosis of Man and Domestic Animals. CRC Press, Boca Raton, Florida, pp. 281-306.

Accepted 19 September 2007 